\title{
PLUG-IN FOR ANALYZING WEB-BASED APPLICATION LOAD TIME TESTING
}

\author{
Shivangi Kaushal \\ Department of Computer Science \\ Punjabi University Regional Centre for Information \\ Technology and Management,Mohali
}

\author{
Jagpuneet Kaur Bajwa \\ Department of Computer Science \\ Punjabi University Regional Centre for Information \\ Technology and Management,Mohali
}

\begin{abstract}
Software testing is very crucial process which helps in finding errors. The aim of web application testing consists of executing the application using combinations of input and state to reveal failures [2]. There are a number of testing techniques which serve different purposes though it is impossible to find and correct all the errors. Software testing of a web based application is done so that the performance and quality of the web based application remain up to the mark so as to meet the user's requirement. In this paper, the stress testing on the web based application is done so as to calculate the load on the web based application. The web based application is put under a lot of stress often to breaking point so that the stability of the web based application could be judged. This is done by attaching the JavaScript code to the web page or the web based application whose stress needs to be tested. The results are stored in the database for future reference.
\end{abstract}

Keywords: Stress testing, Web based application, Functional testing.

\section{INTRODUCTION}

Web applications have grown very large in the last decade. Web applications need to deal with a large number of users as the popularity of web applications have increased tremendously. So it has become important that the users be provided with the best web applications so that it becomes user friendly and gives an easy and fast way to use the application. The development of Web applications is following an evolution similar to that observed for software systems: production is moving from an artistic phase based on highly skilled craftsmen to an industrial phase in which quality is controlled by introducing a structured workflow [13]. A Web application can be considered as a distributed system, with a client-server or multi-tier architecture [2]. So for the above mentioned application testing is carried out.

Software testing is a way to detect errors in the web based application. Software testing is done not only for any one phase of the software development life cycle but it has many areas for testing such as the whole phases of the life cycle such as requirement specification, design, implementation, and maintenance issues. Software testing is done to find faults in the early stage and fix them. Software testing is considered good enough if it is able to remove the faults or errors to the maximum extent from the web based applications. Test Cases are written to validate the testing coverage of the application. These are the documentation part. Test cases are executed and the output is compared with the expected result. Test report is prepared which can be kept for future reference.

The aim of web application testing consists of executing the application using combinations of input and state to reveal failures [2]. Failures can be caused because of any reason either due to the faults in the application or errors done by humans. Web application testing needs models and tools to test the components. For this purpose the basic aspects are required like test models, test levels, test strategies and testing process [2]. In this work, J2EE means Java 2 Platform Enterprise Edition technology is necessary to build large scale, multi-tier applications. The importance of this technology is due to the Java code which is written only once and could be run anywhere when attached with any web based application. This means it has the ability to reuse the components in a web based application.

\section{SOFTWARE TESTING TECHNIQUES}

There are a large number of software testing techniques. These are unit testing, performance testing, regression testing, functional testing, load testing, stress testing, acceptance testing, security testing and many more. In this paper two techniques are discussed, which are functional testing and stress testing.

\subsection{Functional Testing}

Functional testing is a kind of black box testing. The functional testing is done on the functions of the web based application to check their functionality. With the web based applications becoming more complicated and with this the sophistication of these applications also enhances with time, the functionality of the web pages increase due to the different functions such as buttons, links, multiple forms, and many others. This kind of testing emphasizes on the external behavior of the system under test.

The two techniques used for functional testing are Equivalence Class Partitioning and Boundary Value Analysis. Equivalence Class Partitioning is also referred to as partitioning method. The input domain of the program or the test object is partitioned into a finite number of equivalence classes. One single input represents the overall inputs of the sub domain to which that input belongs [15]. Boundary Value Analysis is another much popular technique for black-box testing of a web based application. This type is applicable to the input values that are close to the boundary of the input domain. So the test cases generated are based on the boundary values taken.

\subsection{Stress Testing}

A system stress test refers to tests that put a greater emphasis on robustness, availability and error handling under a heavy load, rather than on what would be considered correct behavior under normal circumstances [3]. Stress testing 
checks the web based applications three main aspects such as response time, throughput, availability and functional integrity. Stress testing is when the web based application is put under extreme conditions which mean stress is put on it beyond normal conditions and it reaches the breaking point of stress. The outcome of stress testing yields performance, reliability, security, quality of the web based application.

\section{PROCEDURE OF STRESS TESTING}

The aim of this research work is to estimate the load time of a web based application. The web application testing tool, which is in actual a client side scripting and is a JavaScript code. This tool is used. This code is attached with the web based application, whose stress testing is to be done. In this a web form is displayed which takes the number of users as the input. Then click on the submit button. Behind the scenes, the plug-in which is attached with the website works. The loadingtime.jsp is called which in turn calls the PageLoadTime.js. It actually calculates the load time of the web based application. After calculation PageLoadTime.js redirects to load.jsp with the load time as an argument. The load.jsp displays load time and makes entry in the database table named "load time". The report is generated in the form of queries in the MySql database. The database table named load_time having three arguments dates, times, openings gives the log report which tells what time is taken to complete the request on which dates. Here we can take parametric values or past project data values from the previously stored database.

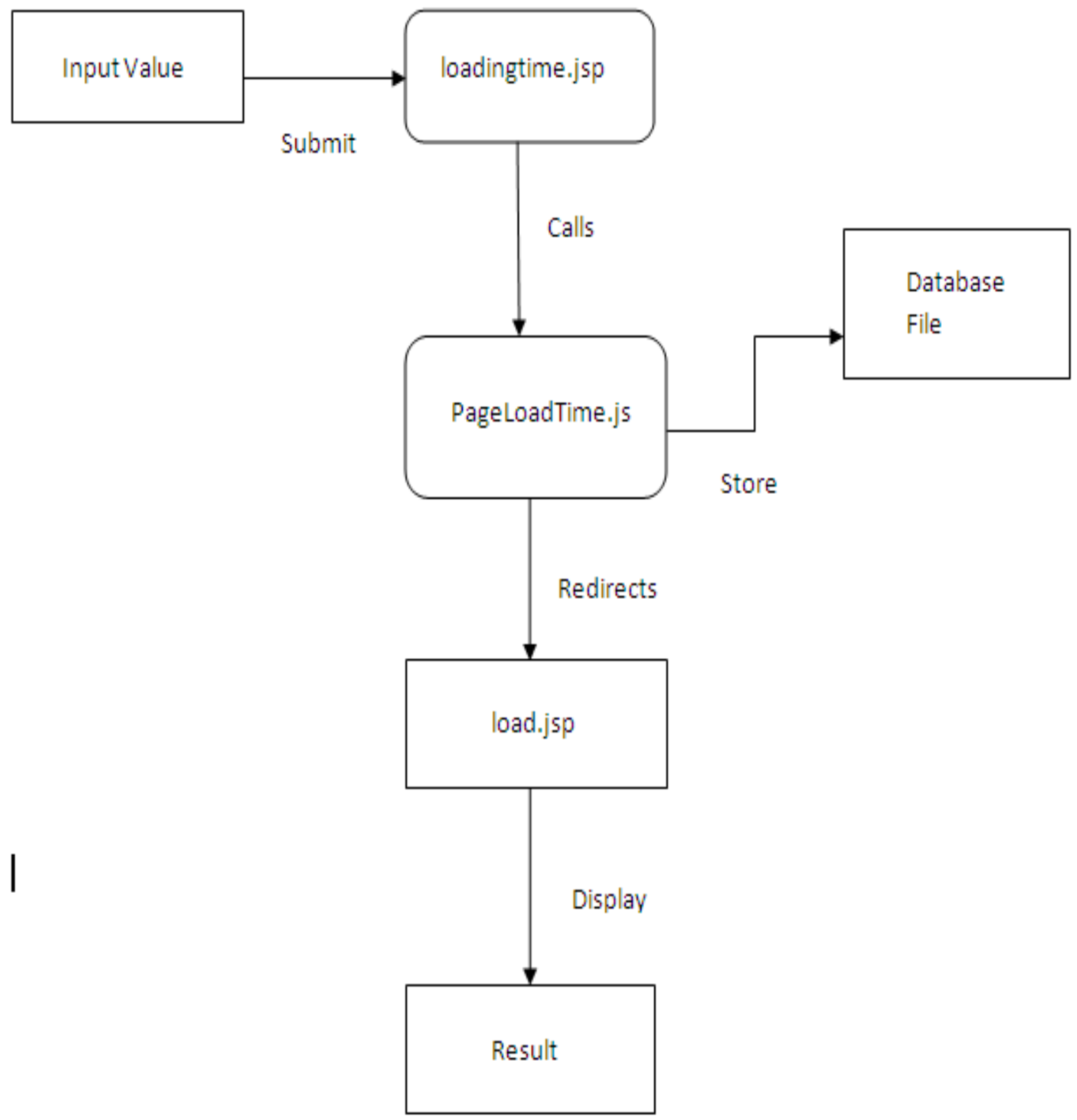

Fig. 1 Represent Diagrammatic View of Stress Testing 


\subsection{Result Analysis}

The snapshots of the above mentioned procedure are shown here.

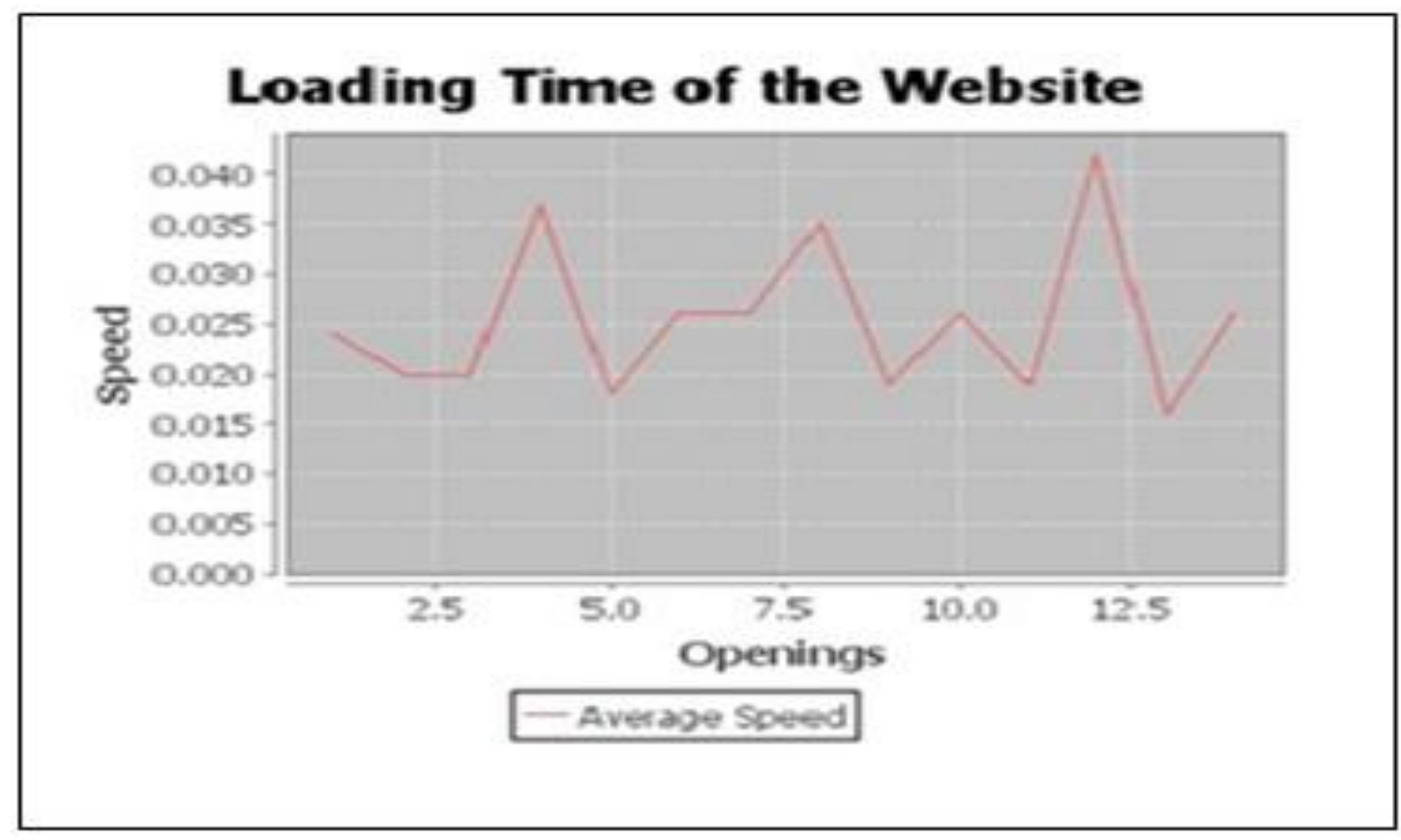

Fig. 2 Load Time Graph

The above shown snapshot is described as here in a way that the graph is generated from the database table which stores the date, time and opening values. The database stores the data when the number of user's hit the web based application page.

The web based applications are used by a number of people and they are on increasing demands due to the internet technology. So testing is done so that the web based application gives good performance and to ensure that the web based application is reliable. Stress testing makes it easy in a way that the database stores the data and the peak point can be checked at any time and the faults can be picked and corrected on time without any delay. In this way the web based application retains its image.

\section{CONCLUSION}

The web based application has been reaching peak range with the advent of the internet technology. So with this the complexity of the web based applications has also increased tremendously with user's increasing demands and to meet their requirements. Therefore, testing of a web based application is vital source to improve the quality, performance, security of the web based application.

Functional testing is done to check the functionality of the web based application's functions such as submit button. Stress testing helps in checking the stability of the web based application by putting stress till the breaking point, which means the point where it crashes when the stress limit gets crossed.In this research work, functional and stress testing has been done. Functional testing is done to check that the functional buttons work properly. Stress testing is done to check the load on the web based application by attaching or plugging the JavaScript code with the web page or the web based application whichever needs to be tested. The result or the outcome based on date, time parameters are recorded in the database. This is a generalized form of testing. These outcomes can be used by the developers for future use when they need to improve the web based application. Thus testing of a web based application plays a crucial role so as to make the application user friendly. In future the work can be extended to large scale web applications with improvements in the application.

\section{REFERENCES}

[1] Charu Babbar and Neha Bajpai, "Web Application Performance Analysis based on Component Load Testing," International Journal of Technology and Applied Science, vol. 2, pp. 22-28, 2011.

[2] Giuseppe A. Di Lucca and Anna Rita Fasolino, "Testing Web-based applications: The state of the art and future trends," in Proceedings of the Twenty-ninth Annual International Conference on Computer Software and Applications, vol. 2, pp. 65-69, July 2005.

[3] Mats Grindal, Jeff Offutt and Sten F. Andler, "Combination of Testing Strategies: A survey," Journal of Software Testing, Verification, and Reliability, vol. 15, pp. 167-199, November 2004.

[4] Chien-Hung Liu, Chih-Tung Hsu et al., "Object-Based Data Flow Testing of Web Applications," in Proceedings of the First Asia-Pacific Conference on Quality Software, pp. 7-16, October 2000.

[5] Giuseppe A. Di Lucca and Massimiliano Di Penta, "Considering Browser Interaction in Web Application 
Testing," in Proceedings of the Fifth IEEE International Workshop on Website Evolution, pp. 74-81, September 2003.

[6] Alessandro Marchetto, Paolo Tonella and Filippo Ricca, "State-Based Testing of AjaxWeb Applications," in First International Conference on Software Testing, Verification, and Validation, pp. 121-130, April 2008.

[7] David Bainbridge, Ian H. Witten et al., "Stress-Testing General Purpose Digital Library Software," SpringerVerlag, vol. 5714, pp. 203-214, 2009.

[ 8] Rudolf Ramler, Edgar Weippl et al., "A Quality-Driven Approach to Web Testing," in Proceedings of the International Conference on Web Engineering, 2002.

[9] Giuseppe Antonio Di Lucca, Anna Rita Fasolino et al., "Testing Web Applications," in Proceedings of the International Conference on Software Maintenance, pp. 310-319, 2002.

[10]Jeff Tian, Li Ma et al., "A Hierarchical Strategy for Testing Web-Based Applications and Ensuring their Reliability," in Proceedings of Twenty-seventh Annual International Computer Software and Applications Conference, pp. 702-707, November 2003.
[11]Lu Luo, "Software Testing Techniques," Carnegie Mellon University, School of Computer Science, Class Report for 17-939A, March 2010.

[12] Sreedevi Sampath, A. Gunes Koru et al., "Prioritizing User-session-based Test Cases for Web Applications Testing," in Proceedings of First International Conference on Software Testing, Verification, and Validation, pp. 141-150, April 2008.

[13]Filippo Ricca and Paolo Tonella, "Analysis and Testing of Web Applications," in Proceedings of the Twentythird International Conference on Software Engineering, pp. 25-34, 2001.

[14]Anneliese A. Andrews, JeffOffutt and Roger T. Alexander, "Testing Web Applications by Modeling with FSMs," International Journal of Software and Systems Modeling, vol. 4, no. 3, pp. 326-345, 2005.

[15]Vineta Arnicane, "Complexity of Equivalence Class and Boundary Value Testing Methods," International Journal of Computer Science and Information Technology, vol. 751, pp. 80-101, 2009. 